\title{
An unusual case of metachronous ascending colon and breast malignancy.
}

\section{Satyajeet Rath*, Rahat Hadi, Niraj Agarwal, Neerja Maurya}

Department of Radiation Oncology, Dr. Ram Manohar Lohia Institute of Medical Sciences, Lucknow, Uttar Pradesh, India

\begin{abstract}
Multiple primary neoplasms are a rare entity and is a growing concern as cancer survivorship is rapidly increasing with timely diagnosis and treatment. The prognosis depends on the stage of the disease and adequate management results in good outcomes. Here, we present a case of dual metachronous malignancy of colon and breast, with the breast primary developing 3 years after the treatment of adenocarcinoma colon. She received right colectomy with ileo-colostomy for colonic malignancy, followed by 8 cycles of adjuvant Capecitabine and Oxaliplatin. The breast malignancy was infiltrating ductal type, pT1N1 and Estrogen receptor positive. It was treated with breast conservation surgery and subsequently, received adjuvant chemotherapy, radiotherapy and is currently on hormonal therapy with Tamoxifen $20 \mathrm{mg}$ daily. A detailed family history and genetic testing should be considered in such cases.
\end{abstract}

Keywords: Multiple primary neoplasms, Metachronous malignancy, Ascending colon.

Accepted on 18 December, 2017

\section{Introduction}

Multiple primary malignancies is a rare phenomenon. There incidence has increased over the last few decades owing to better diagnostic modalities and regular follow-ups and screening [1]. We present case of metachronous ascending colon and breast malignancy.

\section{Case Report}

The patient, a 45 years old female, presented with chief complaints of difficulty in passing stool for one month. It was not associated with blood or mucous in stool, anorexia or weight loss. A thorough evaluation was done. The serum CEA level was 45. Computed tomography was suggestive of carcinoma of the ascending colon (Figure 1). She underwent right colectomy with ileo-colostomy. The post-operative histopathology showed pT2N0, grade II lesion in ascending colon. Following resection, she received 6 cycles of adjuvant Capecitabine plus Oxaliplatin, eight, 3 weekly cycles. She was on follow-up with normal clinical, radiological and serial CEA levels.

Three years after the completion of treatment, she felt a mass in left breast. It was $2 \times 2 \mathrm{~cm}$ in size, hard to touch, non-tender, firm, mobile. It was not associated with any lump in neck or axilla, nipple discharge or retraction, erythema of skin, mass in right breast/axilla of both sides/neck region, retraction of nipple, heaviness in breast, jaundice, headache, pain anywhere in body, seizures, anorexia or weight loss. Mammogram confirmed the same (Figure 2). She was diagnosed as left breast infiltrating ductal carcinoma (IDC), grade II. She underwent left breast conservation surgery with axillary clearance.

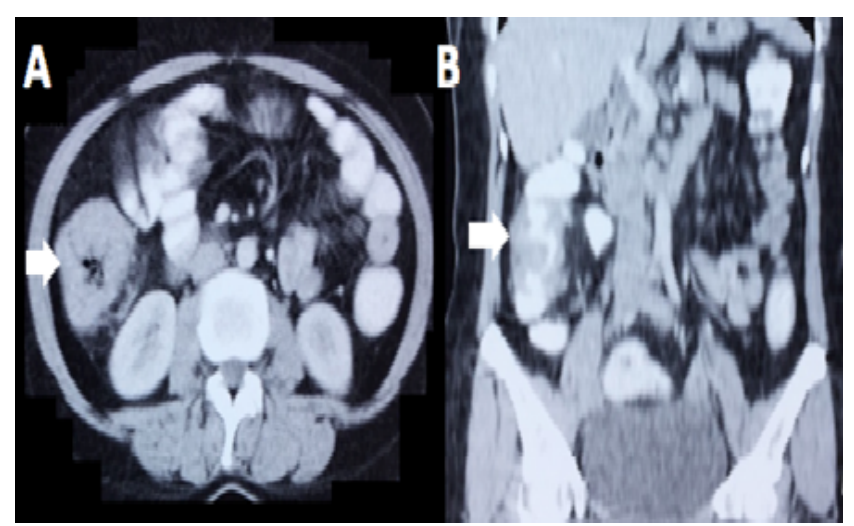

Figure 1. Axial and coronal images of CT Abdomen showing the lesion in right ascending colon.

Post-operative histopathology was suggestive of IDC pT1N1, Estrogen receptor positive, Progesterone receptor positive and HER2neu negative. Then, she received 3 cycles of Adriamycin with Cyclophosphamide followed by 3 cycles of Paclitaxel, $175 \mathrm{mg} / \mathrm{m}^{2}$. Radiotherapy was given as $5000 \mathrm{cGy}$ in 25 fractions to whole breast, axilla and supraclavicular fossa followed by a boost of $1250 \mathrm{cGy}$ in 5 fractions. She has been kept on Tamoxifen daily and was clinically normal on last follow up. 
Citation: Rath S,Hadi R,Agarwal N, et al. An unusual case of metachronous ascending colon and breast malignancy. J Mol Oncol Res. 2018;2(1):11-12.

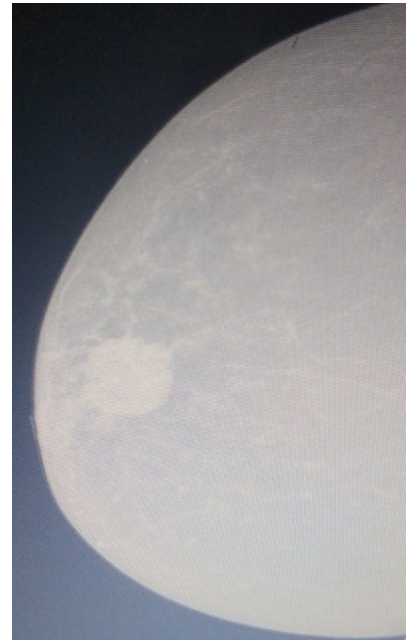

Figure 2. Maommogram showing a $2 \times 2 \mathrm{~cm}$ lesion in the left breast.

\section{Discussion}

Most multiple cancers are double primary cancers. Metachronous malignancies are slowly increasing with improved cancer survivorship over the last few decades. [1] The incidence increases with age [2]. One report by Glicksman et al. [3] showed that over half of all metachronous malignancies arise from four sites-lung, colon, breast and prostate. Another study by Tabuchi et al. [4] reported that the incidence of metachronous malignancies has almost doubled over the last two decades. Pacheco-Figueiredo et al. [5] concluded that the incidence of malignancies is higher in cancer survivors than the general population. The International agencies for research on cancer define multiple primary cancers as those cancers that originally developed in an organ or tissue and are not an extension, recurrence or metastasis. It includes tumours with different topography or different morphology with similar topography [6]. Metachronous tumours are those diagnosed with an interval of $>6$ months between them [7]. Yamamato et al. [8] in a study of 1304 previously treated colorectal carcinoma patients showed that the most common second malignancy in females were gastric and uterine cancer. Other primaries associated are liver, biliary tract primaries, oral cavity, oropharynx, skin, thyroid and lung. High probability of familial syndromes like familial adenomatous polyposis (FAP), hereditary non-polyposis colorectal cancer (HNPCC), BRCA1 and BRCA2 syndromes or Li-Fraumeni disease should be considered in cases with multiple cancers of colon and breast [9]. Long term follow-up with regular examinations and screening should be taken up in patients with colon cancer for early diagnosis of second primary tumour. Survival from the second tumours associated with colon cancer is generally poor as compared a single tumour of the same site [10].

\section{Conclusion}

Incidence of metachronous multiple primary cancers is increasing steadily with improvement in cancer survivorship. A proper history and regular follow-up can help in prompt diagnosis of the second cancer and early treatment, resulting in improved outcomes after treatment.

\section{References}

1. Akasbi Y, Arifi S, Najib R, et al. An Unusual Case of Multiple Primary Carcinomas Breast Cancer and Rectal Adenocarcinoma in a Single Patient Report of a Case and Review of the Literature. Arch Surg Oncol. 2015;1:107.

2. Demandante CG, Troyer DA, Miles TP, et al. Multiple primary malignant neoplasms case report and a comprehensive review of the literature. Am J Clin Oncol. 2003;26: 79-83.

3. Glicksman AS, Fulton JP. Metachronous cancer. R I Med J. 2013;96(4):41-4.

4. Tabuchi T, Ito Y, Ioka A, et al. Incidence of metachronous second primary cancers in Osaka, Japan: update of analyses using population-based cancer registry data. Cancer Sci. 2012;103(6):1111-20.

5. Pacheco-Figueiredo L, Antunes L, Bento MJ, et al. J Cancer Surviv. 2016;10:142.

6. International rules for multiple primary cancers (ICD-0 third edition). Eur J Cancer. 2005;14(4):307-8.

7. Kilciksiz S, Gokce Y, Baloglu A, et al. Characteristics of Synchronous- and Metachronous-Type Multiple Primary Neoplasms A Study of Hospital-Based Cancer Registry in Turkey. Clin Genitourin Cancer. 2007;5:438-45.

8. Yamamoto S, Yoshimura K, Ri S, et al. The risk of multiple primary malignancies with colorectal carcinoma. Dis Colon Rectum. 2006;49:30-6.

9. Iioka Y, Tsuchida A, Okubo K, et al. Metachronous triple cancers of the sigmoid colon, stomach, and esophagus: report of a case. Surg Today. 2000;30:368-71.

10. Rennert G, Robinson E, Rennert HS, et al. Clinical characteristics of metachronous colorectal tumors. Int $\mathrm{J}$ Cancer. 1995;60:743-47.

\section{*Correspondance to}

Satyajeet Rath

Department of Radiation Oncology

Dr. Ram Manohar Lohia Institute of Medical Sciences

Vibhuti Khand, Gomti Nagar

Uttar Pradesh, India

E-mail: satyajeetrath@gmail.com,satyajeetrath@outlook.com 